\title{
Jitter Extraction in a Noisy Signal by Fast Fourier Transform and Time Lag Correlation
}

\author{
Gehan Anthonys $^{\mathrm{a}}{ }^{*}$, Michael J. Cree $^{\mathrm{b}}$, and Lee Streeter ${ }^{\mathrm{c}}$ \\ School of Engineering, University of Waikato, Hamilton 3240, New Zealand \\ aga44@students.waikato.ac.nz, bmichael.cree@waikato.ac.nz, ${ }^{\mathrm{a}}$ lee.streeter@waikato.ac.nz
}

Keywords: fast Fourier transform, noisy signal, periodic jitter, random jitter, time lag correlation.

\begin{abstract}
Jitter in an electronic signal is any deviation in, or displacement of, the signal in time. This paper investigates on decomposition of two types of jitter, namely, periodic and random jitter in noisy signals. Generally, an oscilloscope generates an eye diagram by overlaying sweeps of different segments of a long data stream driven by the reference clock signal. We use the fast Fourier transform with time lag correlation of the signal since we do not have a clock reference signal and apply this technique to simulated noisy signals. We separately injected a random jitter (of known amount), periodic jitter (with known frequency and amount), and both together to various modulation frequencies of sinusoidal signals. The approach is validated by several experiments with numerous values in jitter parameters. When we separately inject random jitter ( $5 \mathrm{ps})$ and periodic jitter ( $5 \mathrm{ps}$ at $4.37 \mathrm{MHz})$ to the signal, we obtained the results ( $4.52 \pm 0.25 \mathrm{ps})$ and ( $4.93 \pm 0.04$ ps at $4.40 \pm 0.04 \mathrm{MHz})$, respectively.
\end{abstract}

\section{Introduction}

The variation in the time of a regular clock signal from its nominal reference is called jitter [1]. In a signal with jitter, the zero-crossing transitions of the cycles are seen to vary in time from the ideal clock timing. Jitter can be characterised, depending on the type of jitter, by parameters such as the amplitude and frequency of the jitter. Jitter amplitude is a measure of the typical time displacement in units of time. If the jitter values are repetitive then the frequency of the repetition is of interest. The time displacement of a digital signal is equivalent to variations in the signal's phase. Controlling jitter is a valuable task because jitter can degrade performance of electronic systems. Measurement of jitter can be performed in the time-domain or frequency-domain. In the time-domain, various techniques and tools such as histogram, bathtub and spectral testing methods are used each with limitations [2-4]. The accuracy of these techniques can only be guaranteed if the jitter measurement is carried out on realtime measuring instruments such as time interval analyzers or high-speed sampling oscilloscopes with very large memory depth to acquire sufficient data. In addition, all these instruments use a sampling clock signal to trigger the time measurement unit to perform the period measurement. Jitter can also be analyzed in the frequency-domain by using the Fourier transform. Then, the energy of the resulted jitter spectrum can be used to calculate the jitter components by application of one of several techniques [5-8]. Some require a jitter-free sampling clock signal (or reference signal) to perform spectral analysis on the signal. As an example, the fast Fourier transform (FFT) and time lag correlation (TLC) functions have been used by Pang et al. (2009) [7] to measure jitter in simulation experiments.

In this paper, we are concerned with measurement of random jitter $(\mathrm{RJ})$ and periodic jitter $(\mathrm{PJ})$ in simulated noisy sinusoidal signals by using FFT and TLC functions. We do not use a reference clock signal and the method is implemented on simulated noisy signals.

\section{Jitter}

Jitter can be considered as consisting of two components: random and deterministic. Random jitter includes all jitter that is random in nature. Typically it as Gaussian distributed, because most noise or jitter in an electrical circuit is caused by thermal noise, which has a Gaussian distribution. Since 
this type of jitter is not bounded, it is characterized by its standard deviation (STD) or root-meansquare (RMS). Deterministic jitter is that component of jitter that can be predicted from prior values. Deterministic jitter is linearly additive and has a non-Gaussian probability density function. It can be characterized by a peak-to-peak value. This type of jitter can be further divided into several types, namely, periodic jitter, bounded correlation jitter and data dependent jitter which includes duty cycle distortion and inter symbol interference [1]. We are concerned only with random jitter and periodic jitter in this paper.

\section{Method and Materials}

For an ideal signal, the cycle period $T$ is defined by the time difference between two consecutive zero crossing points at rising edges of the signal. The jitter $\delta t_{n}$ (see Fig. 1) is the deviation in time of the zero crossing at the $n$th rising edge. Let $t_{n}$ be the actual time of the $n$th rising edge, then the $n$th cycle period is $T_{n}=t_{n}-t_{n-1}$. The jitter is then given by $\delta t_{n}=T+\delta t_{n-1}-T_{n}$.

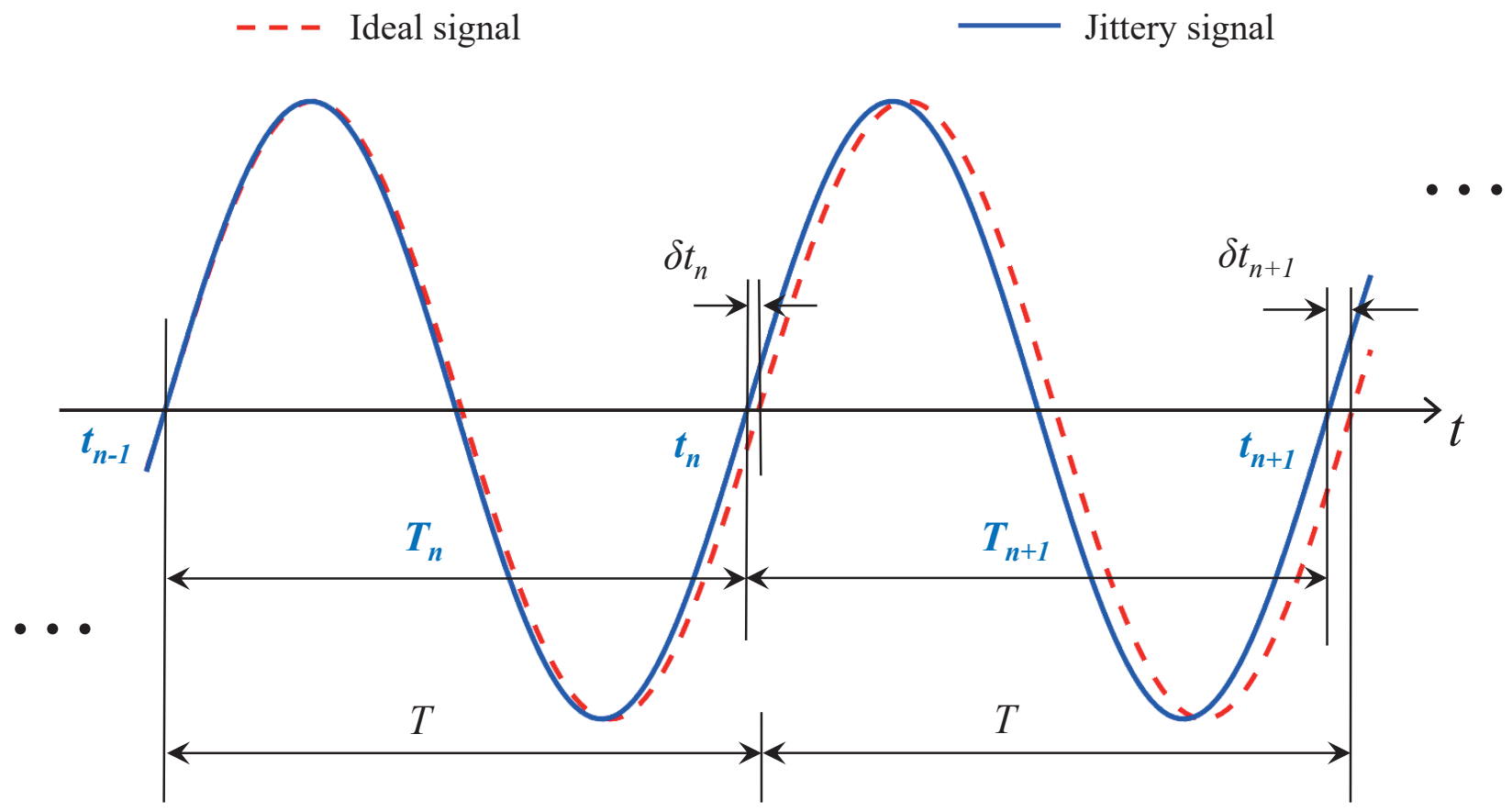

Fig. 1: Jitter definition.

Let the ideal (reference) sinusoidal signal frequency $f$ and amplitude $A$ be

$r(t)=A \sin (2 \pi f t)$.

We assumed zero phase shift and background offset of the signal. The signal with jitter is then,

$$
s(t)=A \sin (2 \pi f(t+\delta t(t))),
$$

where $\delta t(t)$ is the jitter in the signal at time $t$. In this study, we are only concerned with RJ and PJ in the signal. Let,

$$
\delta t^{R J}\left(\sigma_{R J}\right)=\epsilon \sigma_{R J},
$$

where $\epsilon$ is a random variable which is a function of time and $\sigma_{R J}$ is the RMS of the RJ.

Periodic jitter may consist of different frequencies in the signal and these follow the superposition rule. From a signal perspective, periodic jitter is the same as any periodic signal in terms of frequency 
and phase, but its amplitude is jitter in units of timing. The PJ consisting of $L$ different frequencies, is [1],

$$
\delta t^{P J}\left(A_{P J}, f_{P J}\right)=\sum_{l=1}^{L} A_{P J}^{(l)} \cos \left(2 \pi f_{P J}^{(l)} t\right),
$$

where $A_{P J}^{(l)}$ and $f_{P J}^{(l)}$ are the amplitude and frequency of the $l$ th component of the PJ, respectively. In this paper, we consider a single frequency only. Then, the total jitter with RJ and a single PJ is given by

$$
\delta t^{T J}(t)=\epsilon \sigma_{R J}+A_{P J} \cos \left(2 \pi f_{P J} t\right),
$$

and the signal with jitter is,

$$
s(t)=A \sin \left(2 \pi f\left(t+\epsilon \sigma_{R J}+A_{P J} \cos \left(2 \pi f_{P J} t\right)\right)\right) .
$$

Finally, we take the Fourier spectrum of the total jitter in Eq. (5) by using the discrete Fourier transform (DFT) as

$$
F_{k}^{(\delta t)}=\sum_{n=0}^{N-1} \delta t_{n}^{T J}(t) e^{-i \frac{2 \pi n k}{N}}, \quad k=0,1, \ldots, N-1,
$$

where $F_{k}^{(\delta t)}$ is the DFT of the $k$ th sample of the jitter in the samples size of $N$.

\section{Jitter calculation}

A combination of TLC functions and spectrum of the total jitter is used to decompose the jitter parameters, and jitter calculation is performed by considering the energy of the spectrum. Since the autocorrelation function of a variable is only a function of its time lag and not the absolute time, a more general quantification of the jitter is possible by means of the TLC function of the jitter $C_{\delta t}(m)$ with itself over $m$ successive time lags with a sample size of $N$, namely, [9]

$$
C_{\delta t}(m)=\lim _{N \rightarrow \infty} \frac{1}{N} \sum_{n=1}^{N}\left(\delta t_{n+m} \delta t_{n}\right), \quad m \in \mathbb{Z}^{*}
$$

For a signal with random jitter only, Eq. (8) with Eq. (3), simplifies to [4]

$$
C_{\delta t}(m)= \begin{cases}\sigma_{R J}^{2} & m=0 \\ 0 & \text { otherwise }\end{cases}
$$

Likewise, for a signal with single periodic jitter only, Eq. (8) with Eq. (4), simplifies to [4]

$$
C_{\delta t}(m)= \begin{cases}\frac{A^{2}}{2} & m=0 \\ \frac{A^{2}}{2} \cos \left(2 \pi f_{P J} \frac{m}{f}\right) & \text { otherwise. }\end{cases}
$$

To determine $\sigma_{R J}^{2}$ from $C_{\delta t}(0)$ of a signal containing both PJ and RJ, one must find the periodic jitter component first. That can be done with the DFT. Figure 2 is an example of the spectrum of the total jitter $F_{k}^{(\delta t)}$, against its frequency $f^{(\delta t)}$ (i.e., index $k$ ), when injecting both RJ and PJ into the signal. Each point of the spectrum is the square root of energy of that frequency component of the signal. The PJ appears as a clear high peak at its frequency $f_{P J}$. The RJ is spread evenly throughout the spectrum. The line $F_{k}^{(i n t)}$ is the interpolated level of the RJ across that part of the spectrum that the PJ contributes to. This needs to be subtracted from the PJ peak to be able to estimate the energy of the PJ. Then, the other jitter parameters $A_{P J}$ and $\sigma_{R J}$ can be calculated by measuring the energy of the total jitter spectrum where the frequencies contain the signal's energy. 


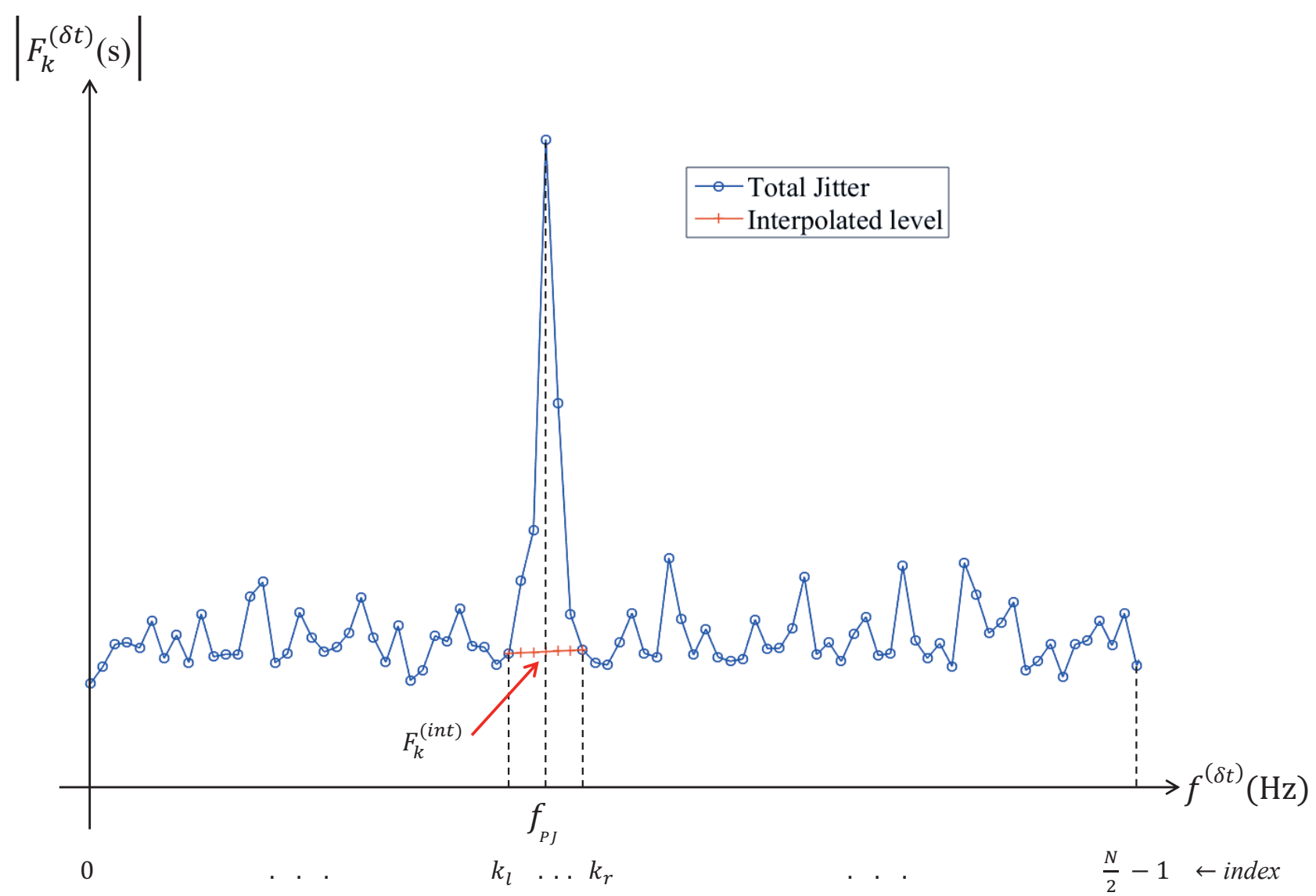

Fig. 2: An example of a spectrum of the total jitter.

This spectrum measures the distribution of energy values as a function of frequency. In the frequencydomain, the energy of a spectral line is calculated by taking the square of the FFT magnitude [10]. Thus, the total energy is given by

$$
\mathrm{E}\left(\delta t^{T J}\right)=2 \sum_{k=0}^{N / 2-1}\left|F_{k}^{(\delta t)}\right|^{2}
$$

where we calculate the summation over one-half the spectrum (because it is symmetric) and multiply by 2 . The total jitter is given by

$$
\delta t^{T J}=\sqrt{2 \mathrm{E}\left(\delta t^{T J}\right)},
$$

where the factor $\sqrt{2}$ is the conversion of the RMS of a sinusoid to its amplitude. The energy of the periodic jitter is in its peak only, therefore

$$
\mathrm{E}\left(\delta t^{P J}\right)=2 \sum_{k=k_{l}}^{k_{r}}\left(\left|F_{k}^{(\delta t)}\right|^{2}-\left|F_{k}^{(i n t)}\right|^{2}\right),
$$

where $k_{l}$ and $k_{r}$ are the left and right indices of the interpolated level of the spectrum, respectively (see Fig. 2). The amplitude of the PJ is

$$
A_{P J}=\sqrt{2 \mathrm{E}\left(\delta t^{P J}\right)} .
$$

Hence by Eqs. (11) and (13), the RMS of the RJ is

$$
\sigma_{R J}=\sqrt{\mathrm{E}\left(\delta t^{T J}\right)-\mathrm{E}\left(\delta t^{P J}\right)}=\sqrt{2 \sum_{k=0}^{N / 2-1}\left|F_{k}^{(\delta t)}\right|^{2}-2 \sum_{k=k_{l}}^{k_{r}}\left(\left|F_{k}^{(\delta t)}\right|^{2}-\left|F_{k}^{(i n t)}\right|^{2}\right)} .
$$




\section{Simulation setup}

We experimented on five simulated signals with various frequencies (i.e. $f_{i}=i \times 10 \mathrm{MHz}, i=$ $1, \ldots, 5)$ and with unit amplitude (i.e. $A=1$ ) throughout the experiments. First, we generated the relevant ideal time-domain signals for each frequency of $f_{i}$. These signals were acquired by sampling with the frequency rate of $F_{s}=8 \mathrm{GSa} / \mathrm{s}$. The number of cycles (number of jitter samples), $N_{i}^{c a l}$ for the five corresponding frequencies $f_{i}$ are given by,

$$
N_{i}^{c a l}=\frac{f_{i} N_{s}}{F_{s}}, \quad i=1, \ldots, 5,
$$

where $N_{s}=2^{16}$, is the number of samples used in the acquired time-domain signals. These calculated number of cycles are not always integer values, therefore spectral leakage appeared in the spectrum of the jitter signal. In order to obtain the spectrum of the total jitter, we chose the number of jitter samples to the nearest integer $N_{i}$ by taking the floor of $N_{i}^{\text {cal }}$ for each simulated signal. Next, the signal with jitter was constructed by injecting a known quantity of jitter for the corresponding timedomain signal. Then, both signals (with and without jitter) were aligned with respect to the first zero crossing point of rising edges and then, the zero crossing points at rising edges for both signals were obtained by using linear interpolation. After that, we calculated the jitter for each cycle by obtaining the corresponding time differences between these zero crossing points of the signal with and without jitter for each experimental simulation. Then, we obtained the spectrum of the total jitter to extract the corresponding jitter parameters for each simulation. In order to control the uncertainties of the results, we used 20 data sets for each simulation setup and obtained the total jitter spectrum for each data set. Finally, we obtain the mean spectrum of the total jitter after taking the magnitude of the spectrum for each data set of the relevant simulation setup.

We consider three types of simulation setup in this study as listed in Table 1. The numerated amount of random jitter, periodic jitter and both types are separately injected into each simulated signal corresponding to the frequency $f_{i}$. We choose the jitter amounts of two, four, six, eight and ten times the lowest value (5 ps) for each of $\sigma_{R J}$ and $A_{P J}$, for the setup denoted as SET. A and SET. B, respectively (see Table 1). In addition, we select a specific value for the frequency of the periodic jitter as $f_{P J}=4.37 \mathrm{MHz}$ throughout the simulations. The combination of these two types of jitter values are chosen as for the setup denoted in SET. C. In addition, by using Eq. (16), the number of jitter samples for each signal frequency are also included.

Table 1: Three types of simulation setup

\begin{tabular}{|c|c|c|c|c|}
\hline \multirow{2}{*}{$\begin{array}{c}\text { Simulation } \\
\text { setup \# }\end{array}$} & \multirow{2}{*}{$\begin{array}{c}\text { Signal frequency } \\
f_{i}[\mathrm{MHz}]\end{array}$} & \multicolumn{2}{|c|}{ No. of cycles } & \multirow{2}{*}{$\begin{array}{c}\text { Injected jitter parameters } \\
\mathrm{RJ} \sim\left(\sigma_{R J}\right) \text { and } \mathrm{PJ} \sim\left(A_{P J}, f_{P J}\right)\end{array}$} \\
\hline & & $N_{i}^{c a l}$ & $N_{i}$ & \\
\hline \multirow{5}{*}{$\begin{array}{l}\text { SET. A : RJ only } \\
\text { SET. B : PJ only } \\
\text { SET. C : RJ with PJ }\end{array}$} & 10 & 81.92 & 81 & \multirow{5}{*}{$\begin{array}{l}\sigma_{R J}=\{5,10,20,30,40,50\}[\mathrm{ps}] \\
A_{P J}=\{5,10,20,30,40,50\}[\mathrm{ps}] \\
f_{P J}=4.37[\mathrm{MHz}]\end{array}$} \\
\hline & 20 & 163.84 & 163 & \\
\hline & 30 & 245.76 & 245 & \\
\hline & 40 & 327.68 & 327 & \\
\hline & 50 & 409.60 & 409 & \\
\hline
\end{tabular}

\section{Results and Discussion}

The spectrum of the total jitter when injecting both RJ and PJ together (denoted as SET. C) for two different values of RMS $\sigma_{R J}$ and $A_{P J}$ for the five simulated signals corresponding to the frequency $f_{i}$ are shown in Fig. 3. 


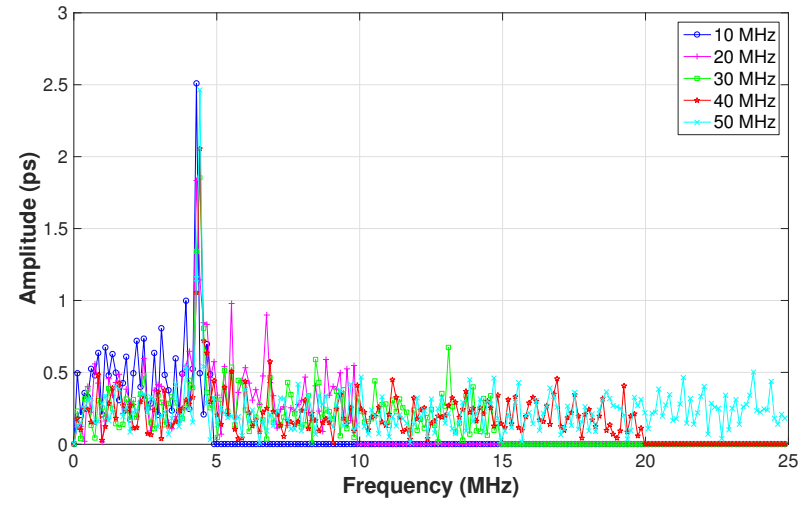

(a)

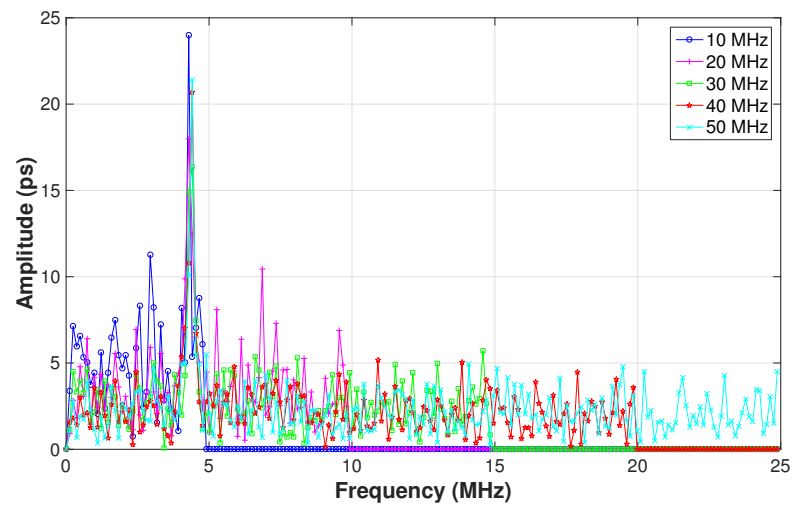

(b)

Fig. 3: Spectrum of total jitter when injecting the RJ and PJ as (a) $\sigma_{R J}=5 \mathrm{ps}, A_{P J}=5 \mathrm{ps}, f_{P J}=4.37$ $\mathrm{MHz}$ and (b) $\sigma_{R J}=50 \mathrm{ps}, A_{P J}=50 \mathrm{ps}, f_{P J}=4.37 \mathrm{MHz}$, for setup denoted as SET. C in Table 1.

In order to illustrate the results in the same plot we padded the amplitude by zeros at the end of first four simulated signals (i.e., for the signal of $f_{i}=10, \ldots, 40 \mathrm{MHz}$ ), to the length of the number of bins of the spectrum of the $50 \mathrm{MHz}$ signal, i.e., 409 (see Table 1). By comparison, we noted that the amplitude level of the spectrum in Fig. 3(b) is approximately 10 times that of Fig. 3(a). It clearly seems the frequency of the PJ (at $4.37 \mathrm{MHz}$ ) is slightly deviated as $4.40 \pm 0.04 \mathrm{MHz}$. We expect this is due to the mismatch between the sampling frequency and the number of cycles (see Table 1) that leads to spectral leakage in the jitter signal. Furthermore, when the spectral leakage presents from a larger signal (amplitude) component, it may significantly overshadow other smaller signals making them difficult to detect [11]. Therefore, the spectrum cannot represent the exact frequency $f_{P J}$ of the $\mathrm{PJ}$, and the energy of the signal is leaked to adjacent bins. As a result, the amount of the $A_{P J}$ of each signal for the similar experiment is not given the same injected value (see Fig. 3).

We compare the actual and measured jitter amounts for each of three simulation setups, separately. Figures 4 and 5 show the comparison of the measured and actual jitter values with different amounts (see Table 1) for 10 and $50 \mathrm{MHz}$ signals for simulations SET. A and SET. B, respectively.

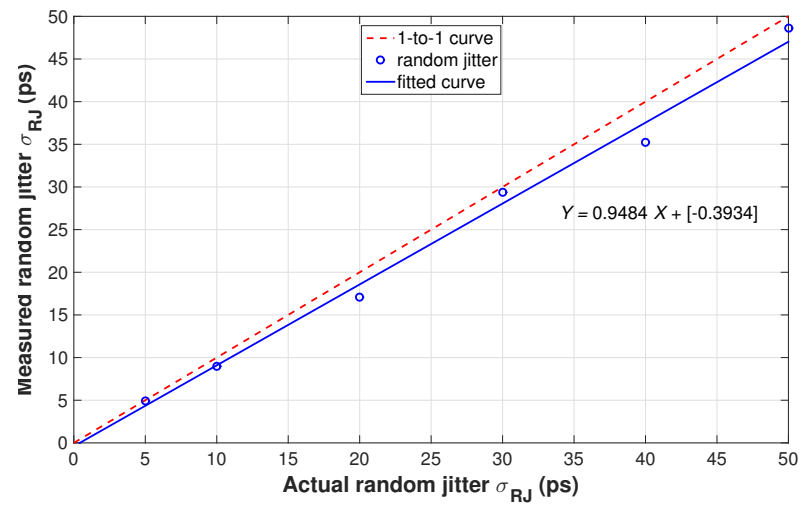

(a)

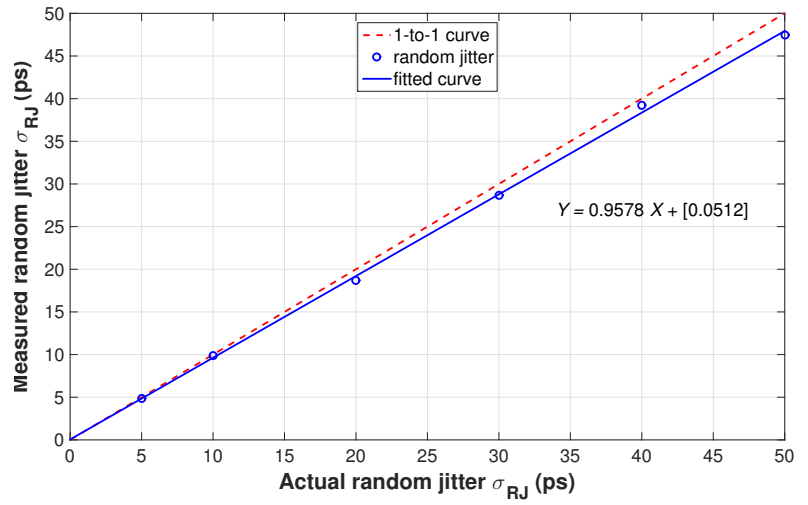

(b)

Fig. 4: Comparison of the actual and measured random jitter in the signal of frequencies, $10 \mathrm{MHz}$ (a) and $50 \mathrm{MHz}$ (b), on the simulation setup denoted as SET. A in Table 1.

In addition, 1-to- 1 curve and the fitted curve through the data points with the relevant linear regression for the measured jitter amounts is also included in each simulation results in the figure. The slope and intercept of this expression are good indicators for the accuracy and offset error, respectively, of 


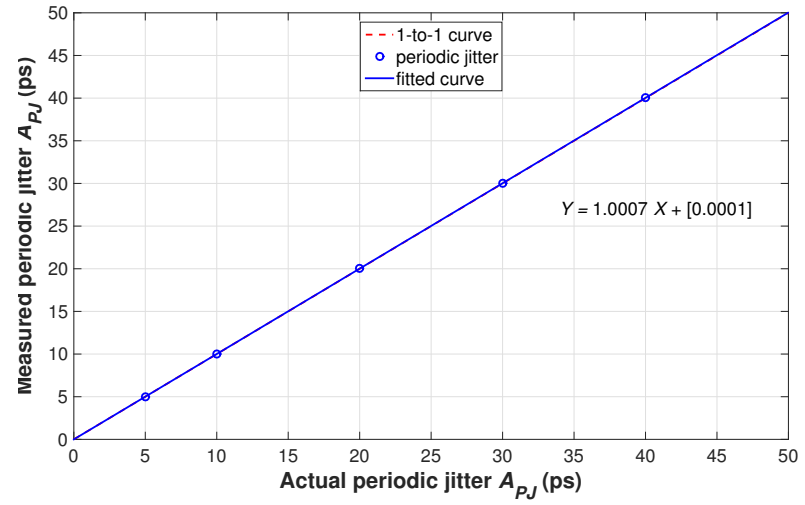

(a)

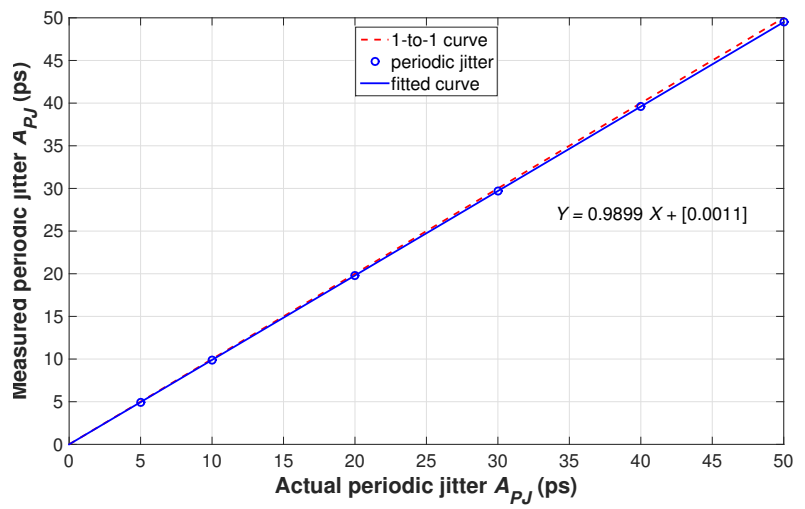

(b)

Fig. 5: Comparison of the actual and measured periodic jitter at $4.37 \mathrm{MHz}$ of the signals, $f_{i}=10 \mathrm{MHz}$ (a) and $f_{i}=50 \mathrm{MHz}$ (b), on the simulation setup denoted as SET. B in Table 1.

the measured jitter parameters. When these two values are closer to one and zero, respectively that indicate the matching of the actual and measured amounts in each simulation. For SET. A, the deviation between the measured and actual values increases (see Fig. 4) when the random jitter amount increases, and note that the offset errors are -0.3934 and 0.0512 for $10 \mathrm{MHz}$ and $50 \mathrm{MHz}$ signals, respectively. We believe this is due to the presence of more zero-crossings with a $50 \mathrm{MHz}$ signal. Statistical uncertainty is reduced due to larger sample size. For the case of PJ (SET. B), the jitter extraction in the absence of any other noise is accurate with very small measurement uncertainty for both $f_{P J}$ and $A_{P J}$.

Similarly for SET. C (both RJ and PJ), on a 50 MHz signal, Fig. 6 shows a strong linear relationship. Fig. 6(a) compares the varies of the PJ with fixed $\sigma_{R J}=5$ ps while Fig. 6(b) compares the varies of the RJ with fixed $A_{P J}=5 \mathrm{ps}$ at $4.37 \mathrm{MHz}$. Among these two plots, the offset error in Fig. 6(b) is relatively larger [0.3165] than the same in Fig. 6(a) [0.2807]. We believe, it is because the PJ affects a particular frequency while the RJ affects all frequencies of the signal with spreading of the energy through whole jitter signal. Therefore, the effect of the RJ on the calculation of PJ is less than the effect of the PJ on the calculation of RJ, when we inject the same amount of each. The measured and actual PJ with fixed $\mathrm{RJ}$ is closer (slope is 0.9745) than the measured and actual RJ with fixed PJ (slope is 0.9404).

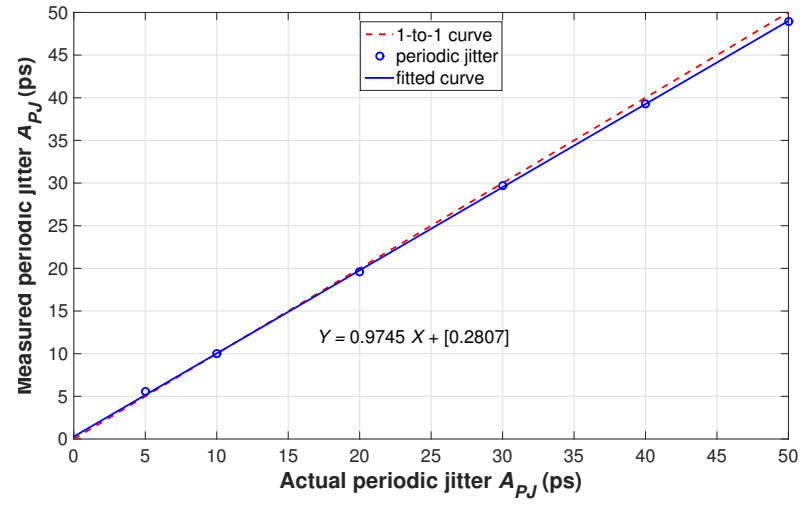

(a)

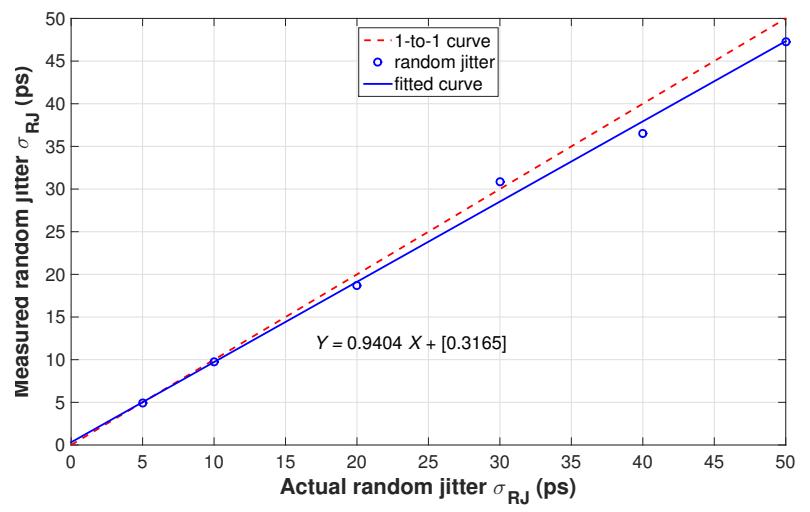

(b)

Fig. 6: Comparison of the actual and measured of periodic jitter with fixed $\sigma_{R J}=5 \mathrm{ps}$ (a) and random jitter with fixed $A_{P J}=5 \mathrm{ps}$ at $4.37 \mathrm{MHz}$ (b), for the signal $f_{i}=50 \mathrm{MHz}$, separately, for setup denoted as SET. C in Table 1. 


\section{Conclusion}

We have investigated an efficient method to extract the periodic and random jitter in simulated noisy signals. First, we aligned the ideal and jittery signals with respect to first zero crossing point of rising edges of two signals. Then, by using an interpolation technique, we obtained the exact zero crossing points at rising edges for each signal. Next, the total jitter is obtained in time domain by calculating the TLC for each cycle and obtaining the spectrum of the same. Finally, by calculating the energy of the frequency spectrum, we extracted the jitter parameters of the signal. We experimented on various frequencies of signals with different values for the parameters of the RJ and PJ. The experimental results show a reasonable accuracy in the jitter measurements. As examples, when we inject only a RJ of RMS values, 5 and $50 \mathrm{ps,} \mathrm{our} \mathrm{approach} \mathrm{was} \mathrm{given} \mathrm{the} \mathrm{values} \mathrm{of} 4.52 \pm 0.25$ and $45.23 \pm 2.44$ ps, respectively. Furthermore, if we inject only the PJ of 5 and 50 ps, separately, at the frequency 4.37 $\mathrm{MHz}$, the calculated parameters were given as $4.93 \pm 0.04 \mathrm{ps}$ and $49.31 \pm 0.46 \mathrm{ps}$, respectively, at the frequency of $4.40 \pm 0.04 \mathrm{MHz}$. If RJ is a large fraction of the total jitter then it can be difficult to separate the PJ accurately. In future work, we plan to use this method for the extraction of jitter components in real-time applications where we have no access to a reference clock signal. This may support to increase the accuracy and precision of the measurements in the system.

\section{References}

[1] M. P. Li. Jitter, Noise, and Signal Integrity at High-Speed, Pearson Education, 2007.

[2] M. Shinagawa, Y. Akazawa and T. Wakimoto, Jitter analysis of high-speed sampling systems, in IEEE Journal of Solid-State Circuits, vol. 25, no. 1, pp. 220-224, Feb 1990. doi: 10.1109/4.50307.

[3] T. Kuyel, Method and System For Measuring Jitter, U.S. Patent 6,640,193.

[4] Q. Dou and J. A. Abraham, Jitter decomposition by time lag correlation. Proceedings of the 7th International Symposium on Quality Electronic Design. IEEE Computer Society, 2006.

[5] T. J. Yamaguchi, M. Soma, L. Malarsie, M. Ishida and H. Musha, Timing jitter measurement of 10 Gbps bit clock signals using frequency division, Proceedings 20th IEEE VLSI Test Symposium (VTS 2002), 2002, pp. 207-212. doi: 10.1109/VTS.2002.1011140

[6] T. J. Yamaguchi, M. Ishida, H. X. Hou, D. Armstrong, K. Takayama and M. Soma, An FFT-based jitter separation method for high-frequency jitter testing with a 10x reduction in test time, 2007 IEEE International Test Conference, Santa Clara, CA, 2007, pp. 1-8. doi: 10.1109/TEST.2007.4437607

[7] H. Pang, J. Zhu, and W. Huang, Jitter decomposition by fast Fourier transform and time lag correlation, International Conference on Communications, Circuits and Systems, Milpitas, CA, 2009, pp. 365-368. doi:10.1109/ICCCAS.2009.5250491

[8] Understanding and characterizing timing jitter. Technical report, Tektronix, Beaverton, OR 97077, United States, 2012.

[9] F. Herzel and B. Razavi. A study of oscillator jitter due to supply and substrate noise. IEEE Transactions on Circuits and Systems II: Analog and Digital Signal Processing 46(1):56-62, 1999.

[10] R. B. Randall, Frequency Analysis, 3rd Edition, Brüel and Kjaer, 1987.

[11] F. J. Harris, On the Use of Windows for Harmonic Analysis with the Discrete Fourier Transform, Proceedings of IEEE, Vol. 66, No. 1, 1978, pp. 51-83. doi:10.1109/PROC.1978.10837 